\title{
Modeling and PID Control of Height Loop of a Kind of Airship Vehicle
}

\author{
Yidong Wang ${ }^{1, a}$, Junwei Lei ${ }^{2, b}$ and Lingling Wang ${ }^{2, c}$ \\ ${ }^{1}$ the $91872^{\text {th }}$ unit of PLA, Beijing, China \\ ${ }^{2}$ Department of control engineering, Naval aeronautical and astronautical University \\ Yanti, 264001 \\ awangyidong96107@163.com, bleijunwei@126.com
}

Keywords: airship; pitch channel; adaptive; PID; stability

Abstract. The airship modeling is the basic of the airship control problem. Based on airship modeling of pitching channel of airship and its nonlinear model, an traditional PID altitude controller of airship is designed. Finally, this control method is proved to be effective by numerical simulation.

\section{Introduction}

From the point of view of foreign application, airship is used as a platform of the early warning and surveillance system. Toshitaka pointed out that stratosphere is very good for airship hovering, because stratosphere is stable[1-3]. So airship become more popular as a relay station communication or monitoring platform. For the above reason, this paper has studied the nonlinear model of pitching channel of stratosphere airship.

The airship modeling is the basic of the airship control problem. And PID control method is a popular method, the PID control method is simple and mature. Based on airship nonlinear model of pitching channel, this paper has studied the PID control of loop of height, and this studies are useful to the further design of airship[4-5].

\section{Model Description}

Based on the previous work, the pitch channel model of airship can be described as follows:

$$
M \dot{x}=f(x)+g(x) u
$$

And $x=\left[\begin{array}{llllll}u & w & q & \theta & x & z\end{array}\right], M$ satisfies

$$
M^{-1}=\left[\begin{array}{llllll}
a_{11} & & a_{13} & & & \\
& a_{22} & & & & \\
a_{31} & & a_{33} & & & \\
& & & 1 & & \\
& & & & 1 & \\
& & & & & 1
\end{array}\right]
$$

The definition of $a_{i j}$ see the definition of $M$ in previous work.

Choose the expect value of all states $u, w, q, \theta, x, z$ are $u^{d}, w^{d}, q^{d}, \theta^{d}, x^{d}, z^{d}$,Define the error variable $e=x-x^{d}, \dot{e}=\dot{x}$, then it hold[6-7]

Use the inverse matrix of $M$

$$
M \dot{e}=f(x)+g(x) u
$$

$$
\dot{e}=M^{-1} f(x)+M^{-1} g(x) u
$$

To make it convenient for reading, some functions can be written as follows 


$$
f(x)=\left[\begin{array}{c}
f_{1} \\
f_{2} \\
f_{3} \\
f_{4} \\
f_{5} \\
f_{6}
\end{array}\right] u=\left[\begin{array}{ll}
u_{1} & u_{2}
\end{array}\right]^{T}
$$

where

$\left[\begin{array}{c}f_{1} \\ f_{2} \\ f_{3} \\ f_{4} \\ f_{5} \\ f_{6}\end{array}\right]=\left[\begin{array}{c}-\left(m+m_{33}\right) w q+Q\left[C_{X 1} \cos ^{2} \alpha+C_{X 2} \sin (2 \alpha) \sin (\alpha / 2)\right. \\ \left(m+m_{11}\right) q u+m a_{z} q^{2}+Q\left[C_{z 1} \cos (\alpha / 2) \sin (2 \alpha)+C_{z 2} \sin (2 \alpha)+C_{z 3} \sin (\alpha) \sin (|\alpha|)\right] \\ -m a_{z} w q(-r v)+Q\left[C_{M 1} \cos (\alpha / 2) \sin (2 \alpha)+C_{M 2} \sin (2 \alpha)+C_{M 3} \sin (\alpha) \sin (\alpha)\right]-a_{z} \sin \theta W \\ q \\ u \cos \theta+w \sin \theta \\ -u \sin \theta+w \cos \theta\end{array}\right]$

Define

$$
M^{-1} f(x)=\left[\begin{array}{c}
f_{a 1} \\
f_{a 2} \\
f_{a 3} \\
f_{a 4} \\
f_{a 5} \\
f_{a 6}
\end{array}\right]=\left[\begin{array}{c}
a_{11} f_{1}+a_{13} f_{3} \\
a_{22} f_{2} \\
a_{31} f_{1}+a_{33} f_{3} \\
f_{4} \\
f_{5} \\
f_{6}
\end{array}\right]
$$

And

Then the system can be written as follows[8-9]

$$
g(x) u=\left[\begin{array}{c}
u_{2} \\
k_{1} u_{1} \\
k_{2} u_{1} \\
0 \\
0 \\
0
\end{array}\right]
$$

$$
\left[\begin{array}{c}
\dot{u} \\
\dot{w} \\
\dot{q} \\
\dot{\theta} \\
\dot{x} \\
\dot{z}
\end{array}\right]=\left[\begin{array}{c}
f_{a 1} \\
f_{a 2} \\
f_{a 3} \\
f_{a 4} \\
f_{a 5} \\
f_{a 6}
\end{array}\right]+\left[\begin{array}{c}
a_{11} u_{2}+a_{13} k_{2} u_{1} \\
a_{22} k_{1} u_{1} \\
a_{31} u_{2}+a_{33} k_{2} u_{1} \\
0 \\
0 \\
0
\end{array}\right]
$$

\section{The PID Control Design For Height Control System}

Inside loop attitude angle controller ensure airship can follow the tracks of expected angle of pitch, so outside loop altitude controller can control altitude[10]. 


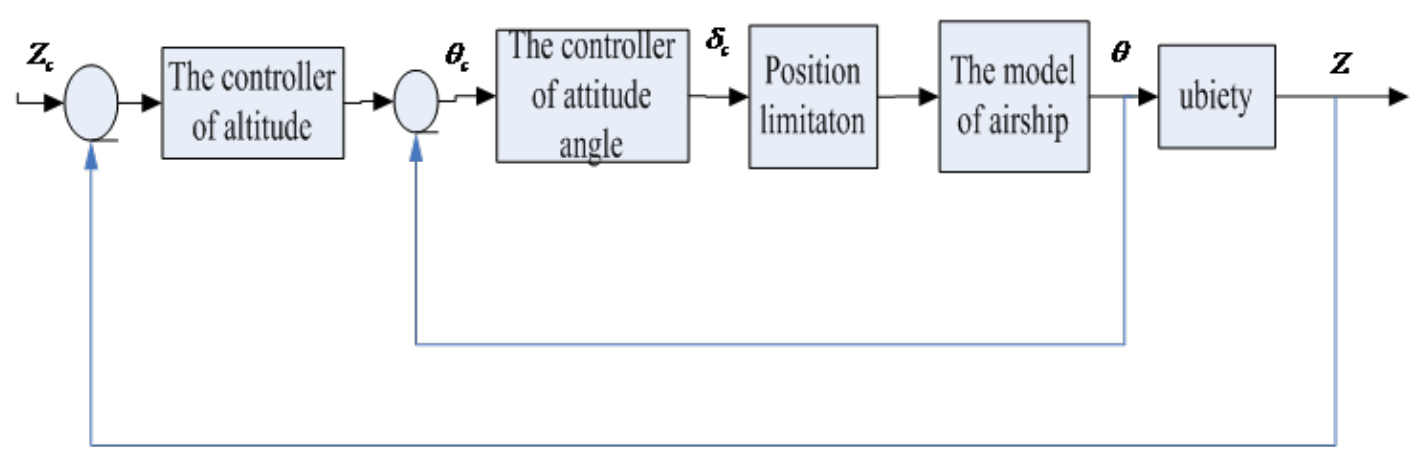

Choose[11-12]

Fig 1 schematic diagram the control loop of altitude

$$
\begin{aligned}
K_{p} & =-0.01 \\
K_{i} & =0 \\
K_{d} & =-0.1
\end{aligned}
$$

\section{Numerical Simulation}

Assume $u=20 \mathrm{~m} / \mathrm{s}, T_{m}=5000 \mathrm{~N}$, initial position $x_{e}=y_{e}=z_{e}=0$. Other quantity of state is 0 , choose $z_{d}=-100$ [13-15], simulation results are as

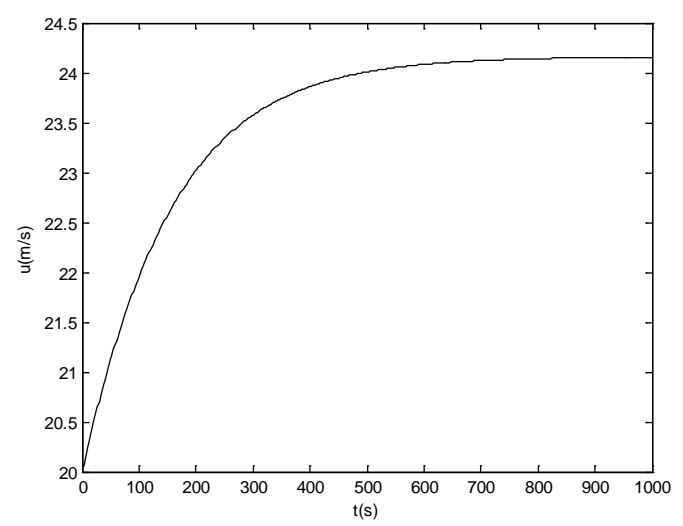

Fig.2 Forward Velocity

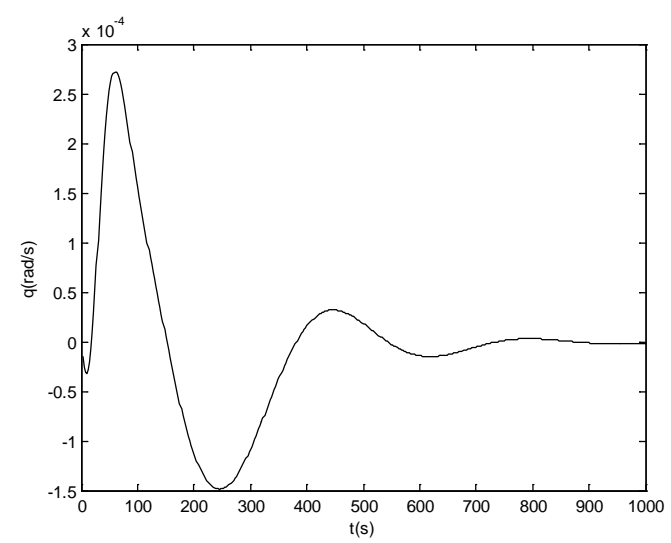

Fig. 4 Angle Velocity

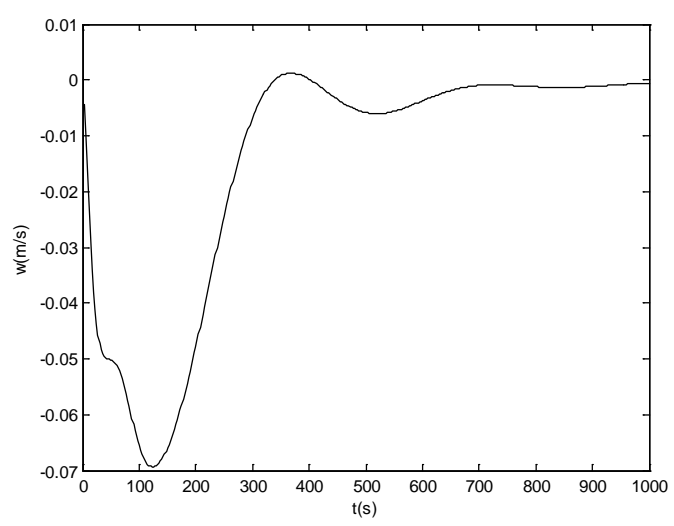

Fig.3 Vertical Velocity

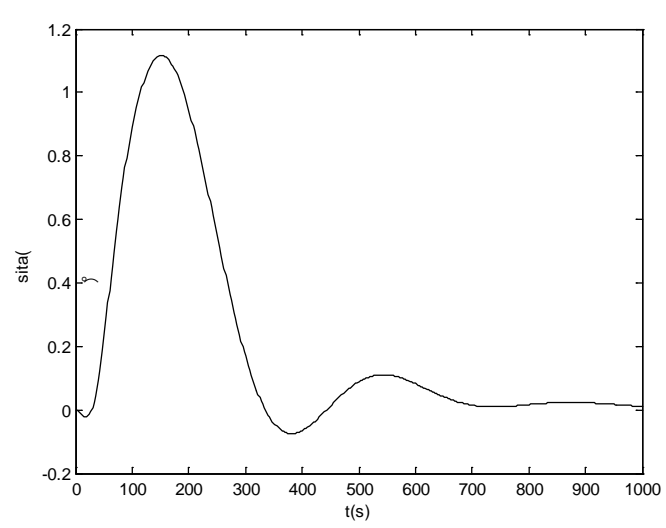

Fig.5 Pitch Angle 


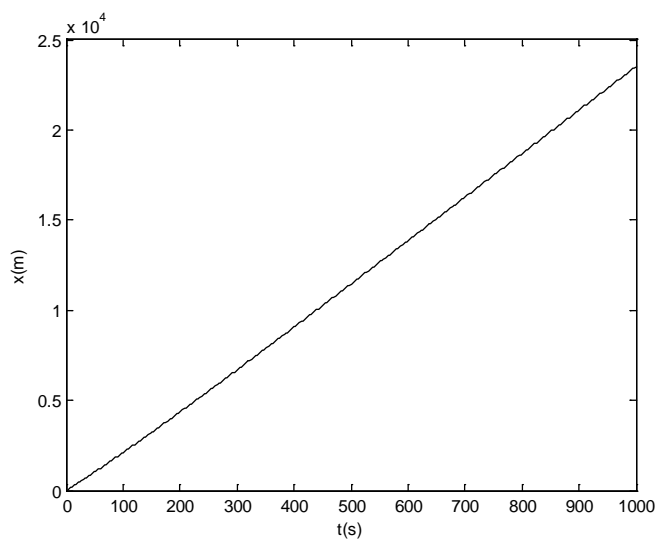

Fig. 6 Flying Distance

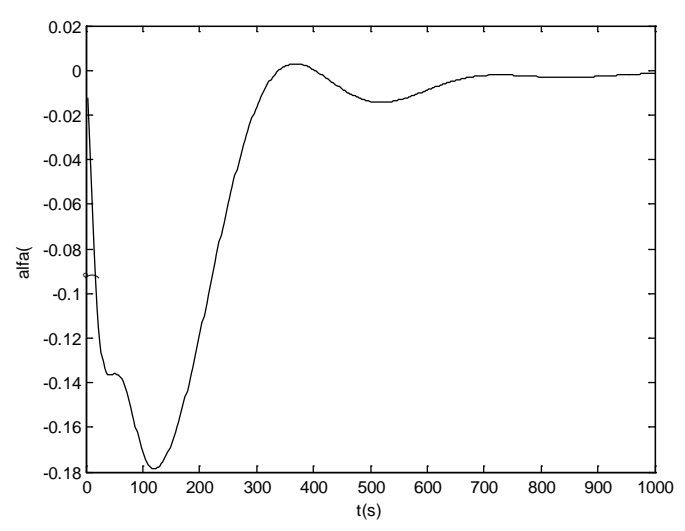

Fig. 8 Pitch Actuator Angle

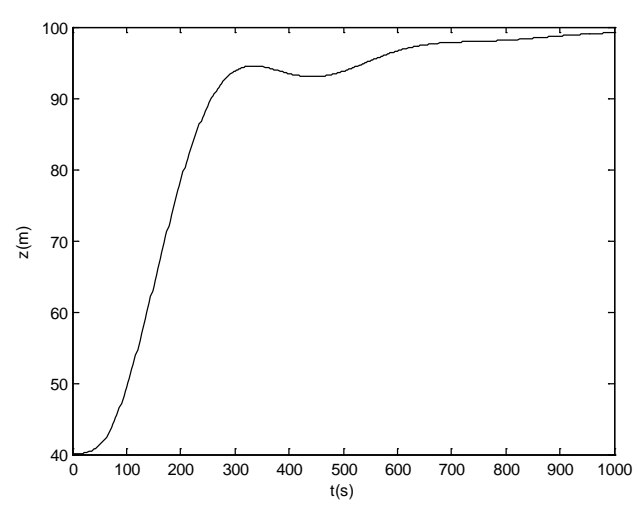

Fig. 7 Height

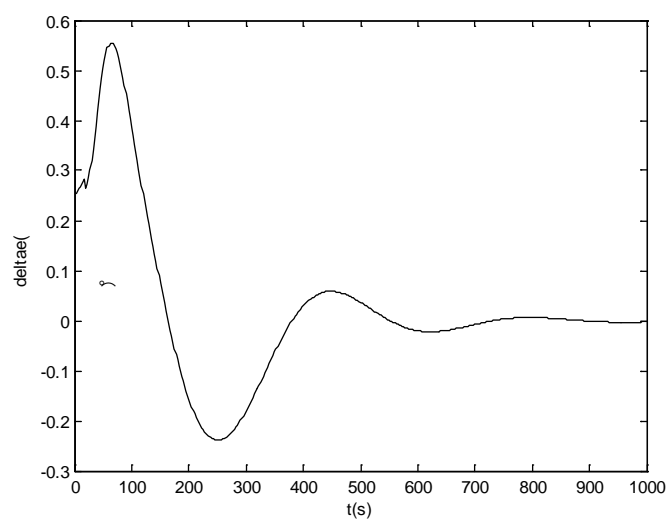

Fig. $9 \delta_{e}$

The simulation results show that airship can follow the tracks of expected altitude in a small area, and be stable.

\section{Conclusion}

The airship modeling is the basic of the airship control problem. And PID control method is a popular method, the PID control method is simple and mature. Based on airship nonlinear model of pitching channel, this paper has studied the PID control of loop of height, and this studies are useful to the further design of airship.

\section{References}

[1] J.S.Uhlman,N.E.Fine,D.C.Kring.Calculation of the Added Mass and Damping Forces on Supercavitating Bodies.The 4th International Symposium on Cavitation,California,2001:7 13

[2] D.Clarke.Calculation of the Added Mass of Elliptical Cylinders in Shallow Water.Ocean Engineering.2001,28(4):61 72

[3] C.J.Atkinson,R.G.Urso.Modeling of Apparent Mass Effects for the Real-Time Simulation of a Hybird Airship.AIAA Modeling and Simulation Technologies Conference and Exhibit,Keystone.2006:21 32

[4] Yokomaku Y.The Stratospheric Platform Airship R\&D Program of Japan. The 2nd Stratospheric Platform Systems Workshop,Tokyo Japan.2000:7 13

[5] S.P.Jones,J.D.Laurier.Aerodynamic Estimation Techniques for Aerostats and Airships.AIAA Lighter-than-Air Systems Conference,Annapolis,2004: 88 94

[6]M. T. Soylemeza, N. Munrob, H. Bakic. Fast Calculation of Stabilizing PID Controllers. 
Automatic, 2003, 39 (7):121 126

[7] Etkin B, Theory of the flight of Airplanes in Isotropic Turbulence Review an Extension,AGARD Rept.1961:372

[8] David K. Schmidt,James Stevens,Jason Roney.Dynamic Modeling,Control, and Station-Keeping Guidance of A Large High-Altitude“Near-Space” Airship.AIAA Guidance,Navigation,and Control Conference and Exhibit. 2006-6781

[9] David K. Schmidt.Modeling and Near-Space Station-Keeping Control of a Large High-Altitude Airship.Journal of Control and Dynamics,2007,30(2):540 547

[10]Azinheira, J.R., A.Moutinho, Hover Control of an UAV With Backstepping Design Including Input Saturations[J]. IEEE Transactions on Control System Technology, 2008.16(3):517-526 